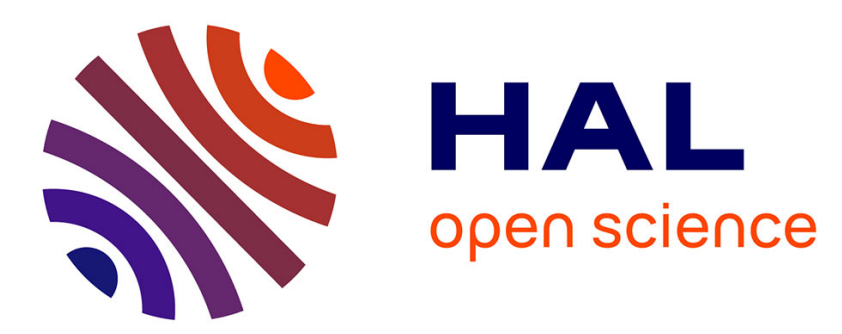

\title{
Magnetic Anisotropy Energy and Interlayer Exchange Coupling in ultrathin ferromagnets: experiment versus theory
}

Klaus Baberschke

\section{- To cite this version:}

Klaus Baberschke. Magnetic Anisotropy Energy and Interlayer Exchange Coupling in ultrathin ferromagnets: experiment versus theory. Philosophical Magazine, 2008, 88 (18-20), pp.2643-2654. 10.1080/14786430802279778 . hal-00514356

\section{HAL Id: hal-00514356 https://hal.science/hal-00514356}

Submitted on 2 Sep 2010

HAL is a multi-disciplinary open access archive for the deposit and dissemination of scientific research documents, whether they are published or not. The documents may come from teaching and research institutions in France or abroad, or from public or private research centers.
L'archive ouverte pluridisciplinaire HAL, est destinée au dépôt et à la diffusion de documents scientifiques de niveau recherche, publiés ou non, émanant des établissements d'enseignement et de recherche français ou étrangers, des laboratoires publics ou privés. 


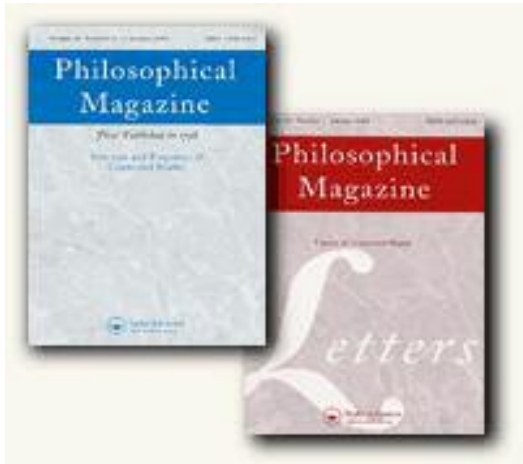

\section{Magnetic Anisotropy Energy and Interlayer Exchange Coupling in ultrathin ferromagnets: experiment versus theory}

\begin{tabular}{|r|l|}
\hline Journal: & Philosophical Magazine \& Philosophical Magazine Letters \\
\hline Manuscript ID: & TPHM-08-May-0134.R1 \\
\hline Journal Selection: & Philosophical Magazine \\
\hline $\begin{array}{r}\text { Date Submitted by the } \\
\text { Author: }\end{array}$ & 13-Jun-2008 \\
\hline Keywords: & magnetic anisotropy, magnetic films \\
\hline Keywords (user supplied): & interlayer exchange \\
\hline & \\
\hline $\begin{array}{l}\text { Note: The following files were submitted by the author for peer review, but cannot be converted } \\
\text { to PDF. You must view these files (e.g. movies) online. }\end{array}$ \\
\hline ICCMS2008.tex
\end{tabular}

\section{ScholarONE" \\ Manuscript Central}




\title{
Magnetic Anisotropy Energy and Interlayer Exchange Coupling in ultrathin Ferromagnets: Experiment versus Theory
}

\author{
Klaus Baberschke* \\ Institut für Experimentalphysik, Freie Universität Berlin, Arnimallee 14, D-14195 Berlin, Germany
}

(June 17, 2008)

\begin{abstract}
To study magnetism and crystallography of nanostructures is one of the most challenging topics, at present. Novel structures were grown, which do not exist in the bulk; the magnetism of these nano-sized particles and films may differ from the bulk by orders of magnitude. Synergistic applications of theory and experiment in materials science are all important for a fundamental understanding. Most important parameters are the Magnetic Anisotropy Energy (MAE) and the Interlayer Exchange Coupling (IEC) in multilayers. We will discuss examples where $a b$ initio calculations adapted to existing experiments disentangle the importance of surface and volume effects in the MAE, as well as a layer-resolved IEC and its T-dependence. The Weinberger-group has unambiguously shown that the "volume part" of MAE is most important to understand the spin reorientation transition (SRT) in Ni/Cu. They also calculated the IEC layer-by-layer in the $\mathrm{T}=0$ limit for a trilayer. Very recently, in theory, spin wave excitation were added to interpret the experimental findings.
\end{abstract}

\section{Introduction}

Magnetism has been a fascinating field for a long time. Traditionally two subgroups have been developed: the single particle magnetism of isolated atoms and molecules and the collective magnetism with ordering phenomena, critical temperature of an ensemble of localized or itinerant magnetic moments. Into the first category belong, for example dilute $3 \mathrm{~d}$, $4 \mathrm{f}$ ions, but also $\mathrm{Cu}, \mathrm{Ag}, \mathrm{Au}$ atoms in the gas phase, they carry a magnetic moment. In an external magnetic field $H_{0}$, they undergo the Zeeman effect, and various experimental techniques (e. g. optical spectroscopy, paramagnetic resonance, etc.) can be used to measure the orbital and spin part of the magnetic moment per particle. The second category focuses on magnetic order, ferro-, or antiferromagnetism. Here the majority of experiments is dealing with hysteresis loops, coercive fields, magnetic domains etc. In this group most of the experimental techniques are limited to temperatures $T<T_{c}$. Spin polarized PE, MOKE, XMCD, they all loose their signal with vanishing magnetization $M$. As a matter of fact, a large fraction of the literature calls the regime above $T_{c}$ "nonmagnetic" instead of "paramagnetic". However both, the para- and ferromagnetic phase, carry the same fingerprint, namely orbital and spin magnetic moments, $\mu_{L}$, and $\mu_{S} . \mu_{L}$ and its anisotropy is the only origin of magnetic anisotropy energy (beside a dipolar contribution). In both phases, for $T \gtrless T_{c}$, this is manifested in the tensor components of the g-factor. In the "subgroup of ferromagnetism" various names have been introduced, like magneto-elastic, magnetostriction, magneto-crystalline anisotropy, etc; they all originate from the same source, the non-vanishing orbital magnetism. Even the so-called anisotropic exchange can be interpreted as hidden orbital magnetism, projected into spin-space. For an isotropic Heisenberg Hamiltonian, $\mathbf{S}_{\mathbf{i}} \cdot \mathbf{S}_{\mathbf{j}}$ it costs no energy to rotate parallel (or antiparallel) aligned spins in space. Only the orbital angular moment $\mathbf{L}$, the non-spherical charge distribution, couples to $\mathbf{r}$-space, the crystallographic lattice.

Fert and Levy $[1,2]$ showed, quite early, that an exchange coupling, for example of Mn-Mn pairs in a dilute $\mathrm{Mn}: \mathrm{Cu}$ alloy, produce no anisotropy, the isotropic exchange interaction cannot explain the fieldcooling memory in spin glasses. Only triangle coupling via an impurity, e. g. Pt or Au, creates a "missing inversion symmetry" along the Mn-Mn axis. They calculated this in $3^{\text {rd }}$ order perturbation theory with dominant spin-orbit interaction at the (Pt or $\mathrm{Au}$ ) impurity site. They also pointed out, that this spin-orbit contribution is the main ingredient in the $3 \times 3$ matrix of coupling with missing inversion symmetry, i. e. the

\footnotetext{
*Corresponding author. Fax +49 30 838-55048. Email: bab@physik.fu-berlin.de 
Dzyakoshinsky-Moriya interaction (DM). The DM interaction, as discussed at this conference, is the lowest order coupling with missing inversion symmetry for the off-diagonal matrix elements - a unidirectional mechanism. It did explain the memory effect in spin glasses and it will explain the exchange bias at interfaces of nanostructures, today. On todays level a $3^{\text {rd }}$ order perturbation theory using Schrödingers equation may not be sufficient - in some cases. One might go right away and solve the full Dirac equation. That however, means that we should not use the picture of Pauli particles, but directly the Dirac particles, with the spin not being a good quantum number. In any case, only the orbital magnetism causes anisotropy in magnetism. Without it, we would have no hard magnets and no magnetic storage media.

Most of the experiments do not measure the anisotropy field (or the interlayer exchange field), but rather the energy. That is to say, the product of $\mu \cdot \mathbf{H}_{\mathbf{a n}}$. Caution must be taken when interpreting these numbers. The magnetic moment at the surface, and at an interface differ significantly from the moment in the inner part of a nanostructure. For example, for a $\mathrm{Co} / \mathrm{Cu}$ (001) film of several ML thickness, the surface layer, facing vacuum has an $\approx 32 \%$ enhanced moment. But at the $\mathrm{Co}-\mathrm{Cu}$ interface the Co moment is $\approx 17 \%$ reduced, due to hybridization effects with $\mathrm{Cu}$ [3]. In this paper we will not discuss the details of the experiment, but refer to recent publications [4-8].

To calculate the MAE from first principle is a challenge. The difference in energy/particle in different crystallographic directions ranges from $\mu \mathrm{eV}$ to few $\mathrm{meV}$, this is a small fraction out of the total energy/atom, being several eV. But, if successful, the theory has great advantage compared to experiments, it can change the crystallographic structure arbitrarily, allows to calculate the magnetism layer-by-layer, can separate orbital and spin magnetism, etc. In the following we will discuss some recent examples, in which the theory has adapted realistic experimental conditions. Both together, experiment and theory, serve for a better fundamental understanding of the MAE (Sec.2) and the IEC (Sec.3) in ferromagnetic nanostructures.

\section{Magnetic Anisotropy Energy (MAE)}

(a)

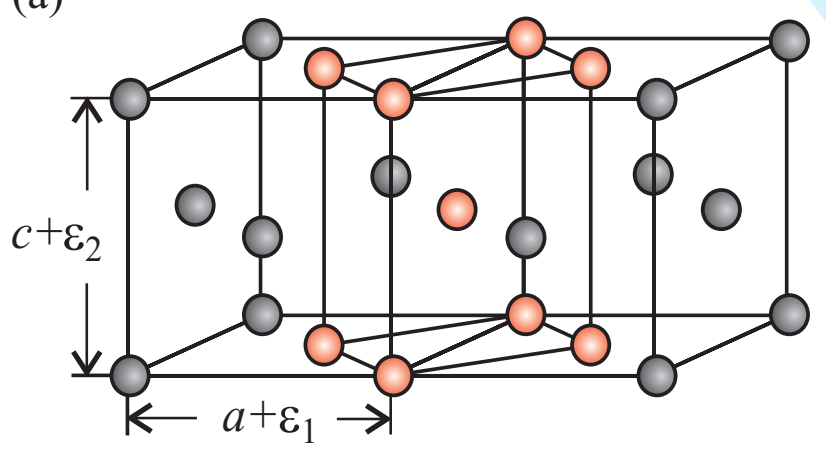

Bain path (b)

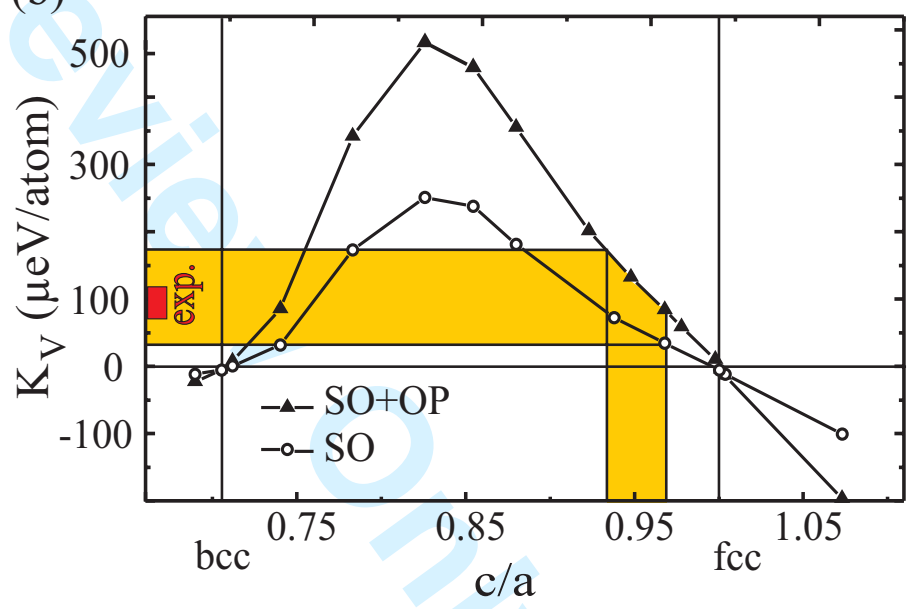

Figure 1. (color online) a) Transformation of an fcc into a bcc structure via the Bain path. b) ab initio calculation of $K_{V}$ for an infinite-sized Ni single crystal, using spin-orbit coupling (open circles), only, and adding orbital polarization (full triangles), also. [9].

The growth of ferromagnetic ultrathin films or nanoparticles opens a complete new variety of crystallographic structures, which do not exist in the bulk. For example, tetragonal $\mathrm{Ni}$ can be grown epitaxially on $\mathrm{Cu}(001)$, or trigonal $\mathrm{Co}$ on $\mathrm{Cu}(111)$. The departure from cubic fcc structure may be small and for some aspects of electronic band structure calculations unimportant. In other words, to assume a perfect cubic lattice for $\mathrm{Fe}, \mathrm{Co}$, or $\mathrm{Ni}$ with the lattice constant of the $\mathrm{Cu}$ substrate crystal facilitates numerical calculation and may be sufficient for some aspects in the band structure and DOS, but for magnetism, the $\mu_{L}$ and the MAE, it is not. Already few hundreds of an $\AA$ change in the n.n. distance may change the MAE by an order of magnitude. This has been nicely demonstrated by the Uppsala theory group [9]. 
They assumed an infinite-sized single crystal of $\mathrm{Ni}$, that is to say, no surface effects or hybridization at the interface are considered; the full MAE originates from the inner part of a crystal, the so called volume part $K_{V}$. The $c / a$ ratio was changed from fcc with $c / a=1$ via tetragonal symmetry to bcc with $c / a=1 / \sqrt{2}$ the Bain path (Fig. 1a).

In experiment only one value can be realized: pseudomorphic growth of $\mathrm{Ni} / \mathrm{Cu}(001)$ creates an fct structure (Fig.2a) with $c / a \approx 0.95$ with $\epsilon_{1}=+2.5 \%$ and $\epsilon_{2}=-3.2 \%$. The lateral n.n. distance in bulk $\mathrm{Ni}$ equals $2.49 \AA$, on $\mathrm{Cu}(001)$ it is $2.55 \AA$, a lateral stretching of $0.06 \AA$, only! In Fig.1b we project this value of $c / a \approx 0.95$ on the theoretical $\mathrm{SO}$ and $\mathrm{SO}+\mathrm{OP}$ calculation (yellow regime), yielding an MAE of $K_{V} \approx 100 \mu \mathrm{eV} /$ atom, in good agreement with experiment [10]. We conclude, very small distortions in the crystal structure can change the MAE by orders of magnitude without employing surface effects, etc. Also in nanostructures and dots, as discussed these days, the crystal structure will depart from the bulk.

(a)

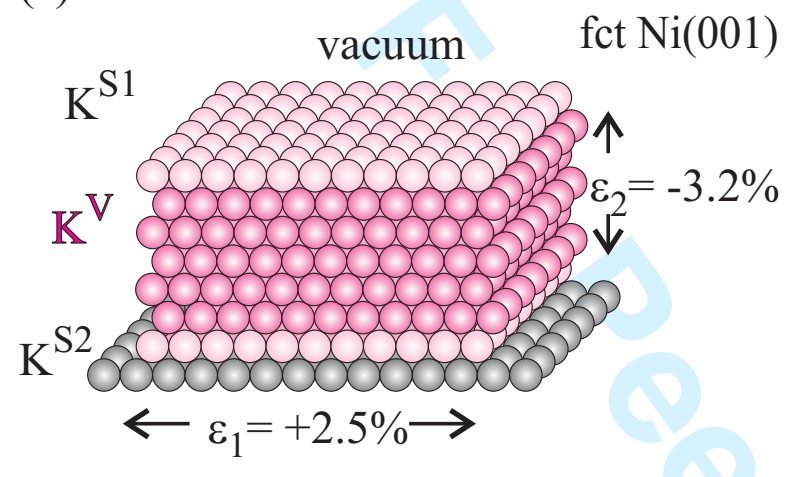

fcc $\mathrm{Cu}(001)$ substrate $\left(\mathrm{a}_{\mathrm{p}}=2.55 \AA\right)$ (b)

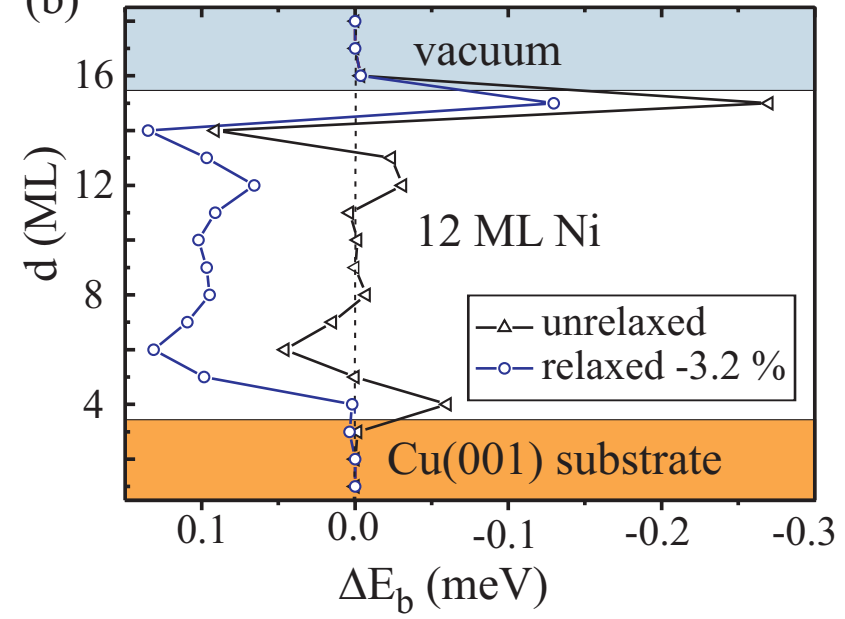

Figure 2. a) Schematic scheme of ultrathin Ni film pseudomorphically grown on $\mathrm{Cu}(001)$. b) ab initio calculation layer-by-layer of K (or $\Delta E_{b}$ ) [11], see text.

In Fig.2a also the surface and interface contributions $K_{S 1}$ and $K_{S 2}$ to the MAE are indicated. Their contribution to the total MAE scales down with the increasing number of layers d (ML) (Néel's argument).In most experiments only the sum of the two is determined.

$$
K=K_{V}+2 K_{S} / d
$$

Here we will discuss only the intrinsic contribution $\mathrm{K}$ (or $\Delta E_{b}$ ) due to the band structure. For the fct $\mathrm{Ni}$ crystal also a second - dipolar - contribution is calculated, but it is very small and will be neglected in the following. In experiments of ultrathin films the dipolar shape anisotropy of $2 \pi M^{2}$ must be subtracted firstly, before discussing the K-value of Eq. (1).

The Weinberger-group has adapted the crystallographic structure of pseudomorphic $\mathrm{Ni} / \mathrm{Cu}(001)$ and calculated the $\Delta E_{b}$ layer-by-layer for several thicknesses of the Ni films [11]. In Fig.2b we show the result for $12 \mathrm{ML}$. It is clear that the surface layer, facing vacuum, carries a large negative anisotropy energy, also the interface layer has a negative contribution. But this effects only one layer, each. The inner part of an unrelaxed (cubic) structure shows more or less no large MAE contribution. But if we accept a tetragonal distorted lattice, we see the same result as in the previous paragraph and in Fig.1: each layer contributes $+\approx 100 \mu \mathrm{eV} /$ layer (open circles in Fig.2b). We conclude: Surface and interface contributions to the MAE may be large and negative, but count only for one layer each. The inner part of a nanostructure, $K_{V}$, will overcome this, because it counts for $n-2$ layers.

Normally experiments cannot measure the MAE layer-by-layer, this can be extracted only from a full set of thickness dependent measurements. When varying the thickness $d$ a second problem enters, due to the finite size effect also $T_{C}(d)$ is a function of thickness (Fig.3a). For example, to measure $K$ and/or 

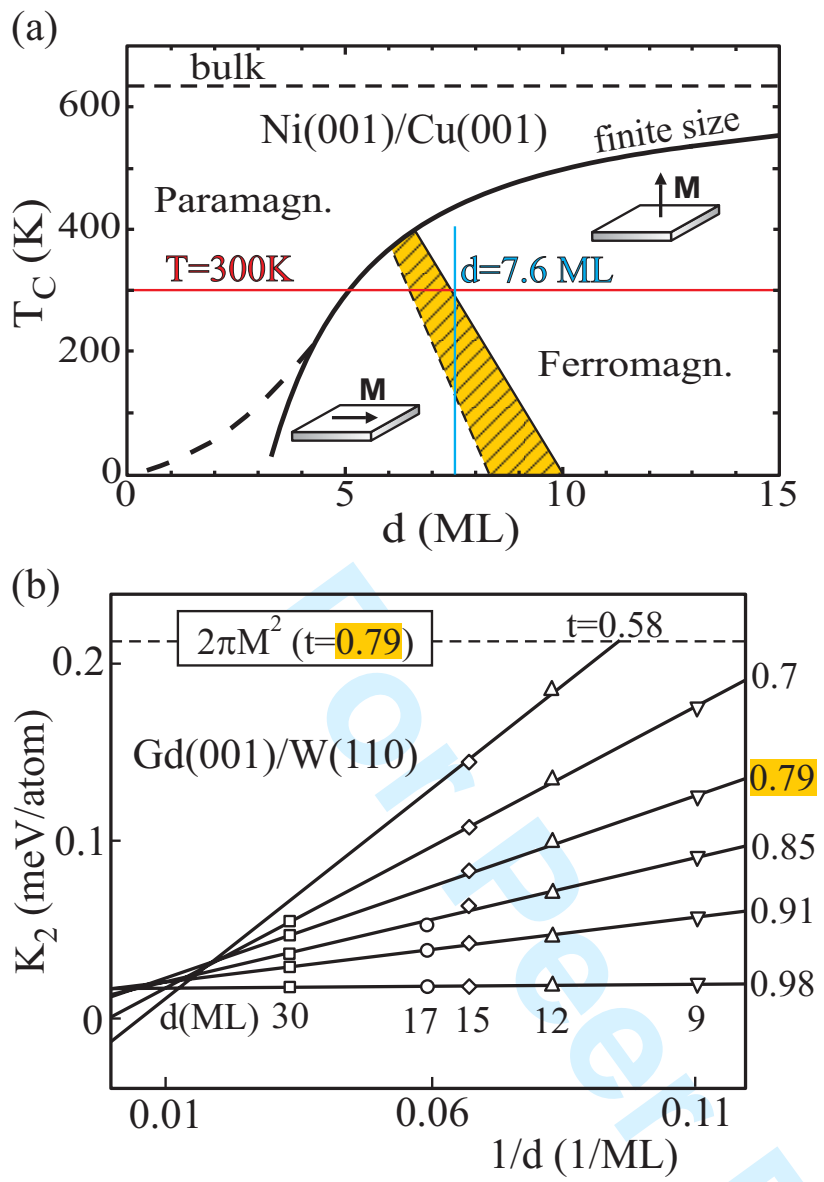

(c)

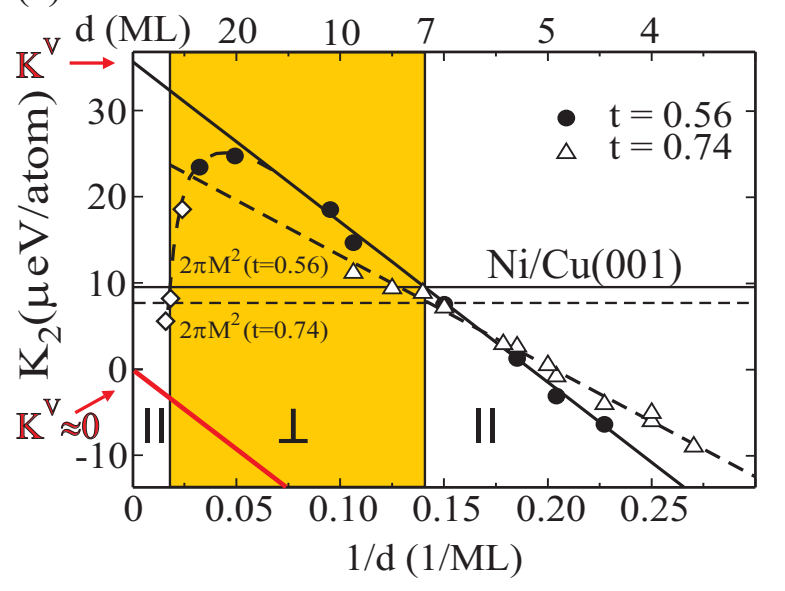

Figure 3. (color online) a) Schematic Curie temperature $T_{C}(d)$ for $\mathrm{Ni} / \mathrm{Cu}(001)$. The solid line indicates the finite size scaling. This is an asymptotic solution for thicker films. Ultrathin films $(d=\leq 4 M L)$ depart from this (dashed line) and remain ferromagnetic at low $\mathrm{T}$. The yellow regime indicates a continuous rotation of the easy axis from in- to out-of-plane [4]. b)Uniaxial anisotropy for Gd/W(110) as function of $1 / \mathrm{d}$ for different reduced temperatures [12]. c) Same plot as in b) for $\mathrm{Ni} / \mathrm{Cu}(001)$

$M$ as $f(d)$ only at a fixed (ambient) temperature, this is of very little use, both $K$ and $M$ are itself a function of $T$, and even more complicated a function of the reduced temperature $t=T / T_{C}$. Both will vanish at $T_{C}$. Fig.3b demonstrates the problem: Gd films at various thicknesses were measured at different reduced temperatures [12]. With such a proper set of experimental data a reliable analysis of $K(1 / d)$ may be performed. Similar results are given for $\mathrm{Ni} / \mathrm{Cu}(001)$ in Fig.3c. Taking $T / T_{C}(d)$ into consideration, we always find a linear 1/d dependence (Eq.1). Quadratic d-dependence has been reported in the literature, that indicates changes in the crystal structure and this may produce all kinds of non-linear $\mathrm{d}$ and $\mathrm{T}$ dependences. As long as we are dealing with a given geometrical structure and want to analyze the thickness- and T-dependence of the MAE, one always expects Eq.1 to be obeyed.

Fig.3c shows the MAE as function of $1 / d$ (dipole contribution is already subtracted from the experimental data). The data range from $d>4 \mathrm{ML}$ to $d<20 \mathrm{ML}$. If normalized to the specific $T_{C}$ at a given $d$ value, the data follow obviously the linear $1 / d$ dependence. All three contributions, $K_{V}, K_{S}$, and $2 \pi M^{2}$ are in the range of 10 to $100 \mu \mathrm{eV} /$ atom, that is to say, surface and volume MAE are in the same order of magnitude. Here the physics of SRT becomes very transparent: $K>2 \pi M^{2}$ favors out-of-plane, but if the dipolar energy wins, in-plane is the easy axis, certainly. So, let's ask the question, why happens a SRT for Ni , but not for $\mathrm{Fe}$ and $\mathrm{Co}$ ? We see in Fig.3c that the dipolar contribution increases quadratic with $M$, i.e. for $\mathrm{Fe}$ and Co the horizontal $2 \pi M^{2}$ line moves up by a factor of 8 to 14 and will never intercept with Eq.1. So, the small magnetic moment of Ni keeps the shape anisotropy low and the positive K-anisotropy may overcome this. Secondly, what causes the SRT, $K_{V}$ or $K_{S}$ ? Commonly it is argued in the literature, that the surface contribution is responsible. Here we show, that this is not the case: $K_{S}$ is negative with a negative slope of $100-200 \mu \mathrm{eV} /$ atom, like for many other systems (see next paragraph). But important is the intercept of $K(1 / d)$ at the $\mathrm{y}$-axis. For bulk fcc Ni $K_{V} \approx 0$ (red line) and $\mathrm{K}$ would never exceed $2 \pi M^{2}$, 
assuming the same negative slope. Only the large intercept of $K_{V} \approx 35 \mu \mathrm{eV} /$ atom for the perturbed fct structure moves the linear 1/d dependence up and causes an SRT transition between 7 to 10 ML. Finally, we see at $\approx 20 M L$ the experimental data depart from Eq. 1 . At this thickness the Ni film "remembers " its bulk fcc structure and the crystal relaxes back to cubic symmetry with a reduced MAE and many misfit dislocations.

Ultrathin Co films on $\mathrm{Cu}$ have been investigated by many groups with high intensity. In the beginning of the nineties the Philips group as well as Gradmann and coworkers measured the uniaxial anisotropy $K_{2}$ of $\mathrm{Co} / \mathrm{Cu}(111)$, shown as diamonds and squares in Fig.4a [13,14]. At first glance it looks, as if $K_{2}$ increases as function of $1 / d$ with a positive slope. But these data were taken at fixed (ambient) temperature and the thickness dependence of $T_{C}$ was ignored. Farle et al. [15] remeasured and plotted the MAE at constant reduced temperature in Fig.4b. For ultrathin thickness of $d \leq 6 \mathrm{ML}$ we see a linear $1 / d$ dependence. In this regime the Co layers grow pseudomorphically on $\mathrm{Cu}(111)$. This produces a small trigonal $(a=b=c$ but $\alpha=\beta=\gamma \neq \pi / 2$ ) distortion in the cubic lattice. If this perturbed cubic structure would grow up to infinite thickness, the intercept at the y-axis is about $K_{V} \approx 95 \mu \mathrm{eV} /$ atom. This is very close to the bulk value for hcp Co. In reality the pseudomorphic growth stops at about $6 \mathrm{ML}$ and we turn back to the standard fcc Co with small MAE. The arrows in Fig.4a indicate, that the data measured at $300 \mathrm{~K}$ will move down to negative values, when taking the change of $T_{C}(d)$ into account. In summary: To the best of our knowledge, we find in the literature always $K(d)$ data following Eq.1. Furthermore we believe, that this type of "K-analysis" is one of the most sensitive techniques to detect small structural changes. It may be more sensitive than LEED or XRD. If other thickness dependent behavior for the MAE is reported (for example quadratic, see e. g. [16]) this will be caused by structural changes as function of d or T. But then, all kinds of functional dependencies may happen, even discontinuities.

(a)

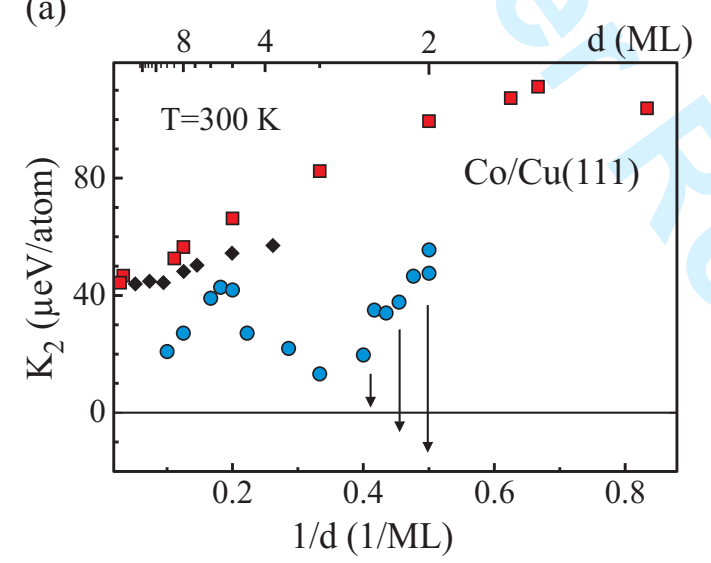

(b)

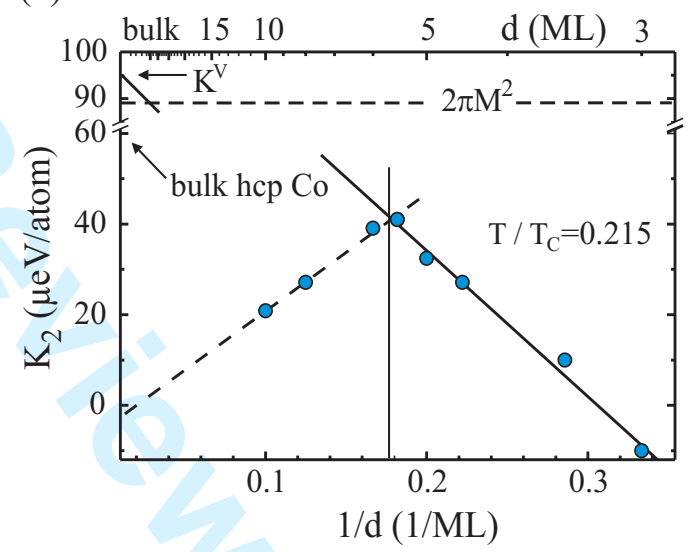

Figure 4. (color online) Uniaxial anisotropy for $\mathrm{Co} / \mathrm{Cu}(111)$ as function of $1 / d$, a) for a fixed temperature of $T=300 K$ and b) for a constant reduced temperature. Squares and diamonds are taken from [13,14] and circles from [12].

Finally, we want to discuss the combined effort of theory and experiment to understand the manipulation of the surface anisotropy $K_{S}$. The $\mathrm{Ni} / \mathrm{Cu}(001)$ system has been investigated by several groups, it was exposed to H, O, or CO gas $[17,18]$. These authors reported a shift in the SRT moving from $\approx 11 \mathrm{ML}$ to thinner values of about $7 \mathrm{ML}$, depending on the gas adsorption. In [19] the Ni film was measured, facing vacuum, being caped with $\mathrm{Cu}$, and being grown with oxygen as surfactant. The experimental results are shown in the inset of Fig.5. $K_{2}$ follows the linear $1 /$ d dependence in the range of 5 to 12MLs with different slopes. That is not surprising, we expect only a change of the surface contribution, and indeed all 3 lines can be extrapolated to the same intercept at the y-axis of $K_{V} \approx 20$ to $25 \mu \mathrm{eV} /$ atom. We also see that the reduction of $K_{S}$ is moderate for $\mathrm{Cu}$ capping, and the strongest for oxygen surfactant growth. That is explained by $\mathrm{Wu}$ and coworker [19] and displayed in Fig.5. They calculated the magnetic anisotropy energy for clean $\mathrm{Ni}_{n}$ slabs with both sides vacuum, for $\mathrm{Cu} / \mathrm{Ni}_{n} / \mathrm{Cu}$ superlattices, and for a $c(2 x 2)$ oxygen adlayer on $\mathrm{Ni}_{n}$ films. $n$ ranges from 5 to 15 layers. For the oxygen surfactant growth the self consistent calculation results in an outward relaxation of the top Ni layer, and a buckling of the second layer. In principle, this was known from the early research of the Ni surface, but here also the MAE of this layer was 


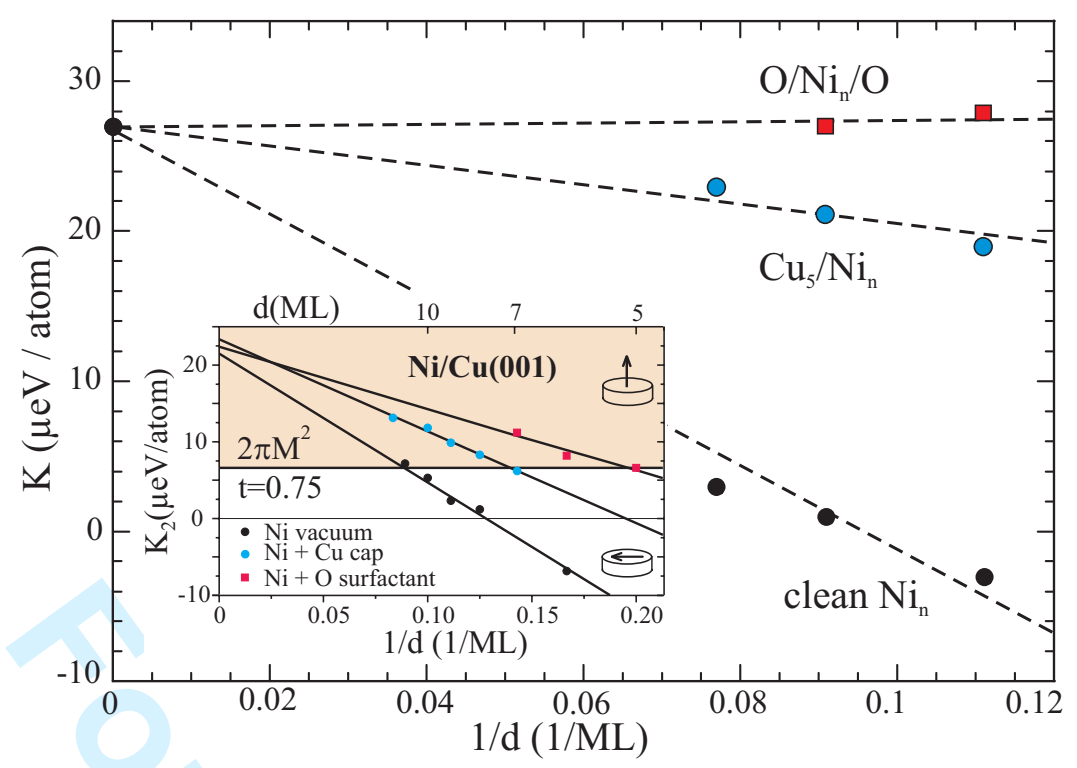

Figure 5. (color online) Calculated MAE for clean Ni films, $\mathrm{Cu} / \mathrm{Ni} / \mathrm{Cu}$ superlattices, and Ni films with a c(2x2) oxygen adlayer [19]. The inset shows the corresponding experiments. For details see text.

calculated and it turned out to be almost zero, $K_{S 1} \approx 0$. Such a calculation gives also a detailed insight in the electronic band structure and how this is effected by oxygen. There is almost no change in the number of $d$ electrons in the surface layer, there is no formation of $\mathrm{NiO}$ on the surface, but one finds a pronounced antibonding O-Ni peak on top of the Ni $d$ band, i. e. an O-induced surface state with $d_{x z}$ character. Its SO splitting is the main feature which reduces $K_{S 1}$. This combined experimental and theoretical effect, first of all, results in a fairly good agreement. Secondly and more important, the calculated spin dependent electronic band structure can explain, what causes what.

\section{Interlayer Exchange Coupling (IEC) and its temperature dependence}

The second, equally important, magnetic parameter in ultrathin multilayers is the IEC. This interaction is known to oscillate between ferro- and antiferromagnetic alignment of two ferromagnetic layers, separated by a nonmagnetic spacer. A vast number of review papers are available, we refer to Chapters in [8]. When measuring or calculating the free energy, both, the MAE and the IEC, enter and it is not always easy to disentangle these. The experimental procedure in FMR measurements opens one way to determine the MAE $(K)$ and IEC $\left(J_{\text {inter }}\right)$, separately. In an in situ UHV-FMR experiment firstly a single film is measured, and the $K$-value determined, then the second FM film is evaporated and the only leftover parameter to be determined is $J_{\text {inter }}$, for details see Ref. [7]. Usually this is measured at finite temperatures and needs to be extrapolated to $T=0$, when comparing with $a b$ initio calculations .

Again, the Weinberger-group has adapted a realistic experimental situation and calculated $K^{j}$ and $J_{\text {inter }}{ }^{j}$ layer-by-layer for a prototype system of a $\mathrm{Ni}_{8} / \mathrm{Cu}_{j} / \mathrm{Ni}_{9}$ trilayer [20]. The results are shown in Fig.6. The $K$-values are different for 8 and 9 ML of Ni (Fig. 6 a and c) and strongly positive when the relaxation of $-3.2 \%$ is taken into account (see Section 2). In Fig.6 b and d the IEC per layer is plotted. First of all, we see that for $3 \mathrm{ML}$ of $\mathrm{Cu}$ the IEC is negative (AFM coupling) and positive (FM coupling) for $9 \mathrm{ML}$; in agreement with experiment. The main contribution to the IEC originates from the first Ni layer at the $\mathrm{Ni} / \mathrm{Cu}$ interface, but also the adjacent $\mathrm{Cu}$ layers contribute - see Fig.6 b. We recall that at a $\mathrm{Ni} / \mathrm{Cu}$ interface $\mathrm{Cu}$ carries an induced magnetic moment. Both, $\mathrm{Ni}$ and $\mathrm{Cu}$, have small but finite orbital moments. This $\mu_{L}$ is the source, which couples - via SO interaction - the spin to the crystallographic lattice - we will come back to this. The absolute value of the calculated IEC of approximately 40 to $150 \mu \mathrm{eV}$ is difficult to be compared to the experiment, because also the measured value is model dependent. In the analysis of the FMR data a "macroscopic" Heisenberg Hamiltonian is used, and that $J_{\text {inter }}$ is not the same as a "microscopically" layer wise calculated IEC in Ref. [20]. 


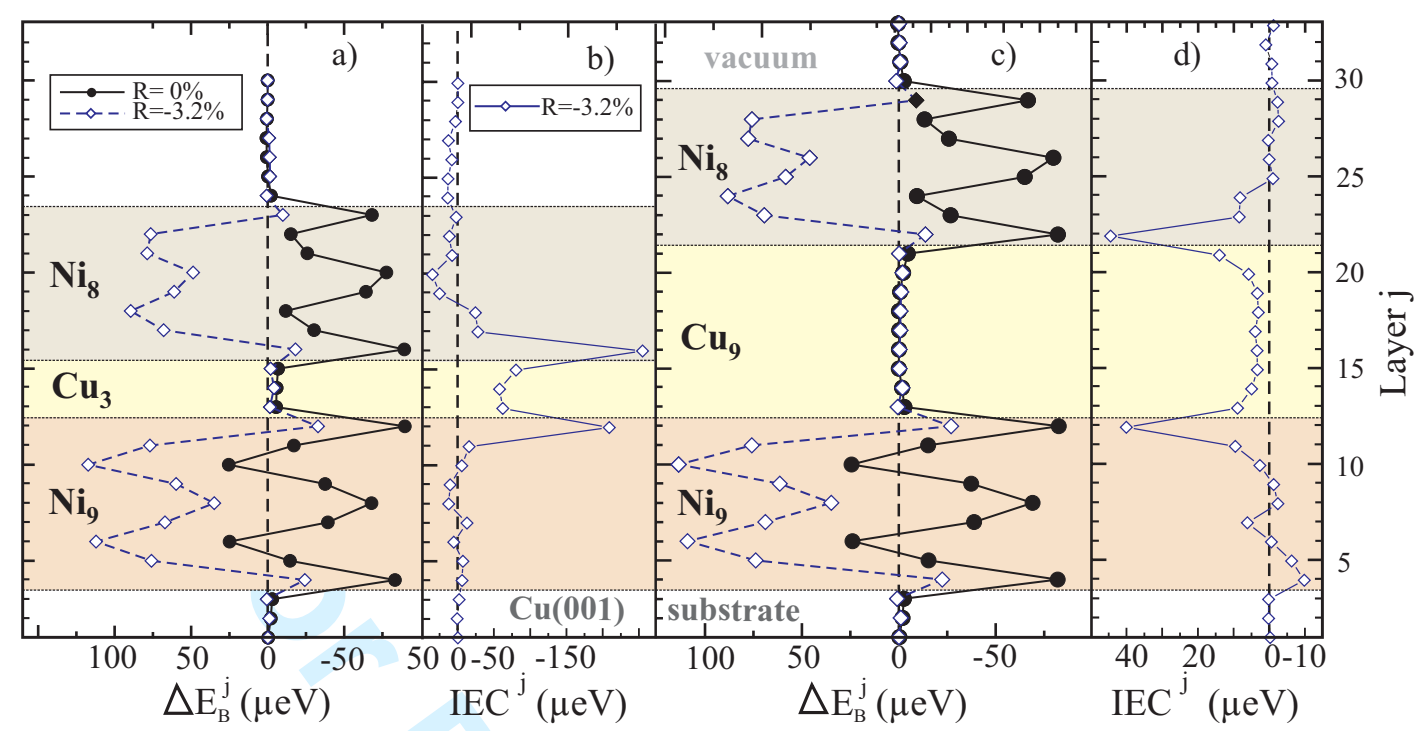

Figure 6. (color online) Calculated layer-resolved MAE (or $\Delta E_{b}$ ) and IEC for a Ni/Cu/Ni trilayer pseudomorphically grown on a $\mathrm{Cu}(001)$ substrate. The number of the Ni layers is fixed to $j=8$ and 9 . The $\mathrm{Cu}$ spacer thickness equals $j=3$ in a) and b) and $j=9$ in c) and d). Taken from [20].

In earlier review articles $T=0$ calculations have been compared with experiments measured, let's say, at room temperature. That may be justified for thicker Fe and Co films, having almost bulk $T_{C}$. For ultrathin films and in particular for $\mathrm{Ni}$ one needs to ask the question: What causes the T-dependence of the IEC, is it mainly an electronic band structure effect, smearing of the Fermi edge, or are spin wave excitations more important, which depend on $T_{C}$ ? Both theoretical models have been proposed. In Ref. [21] electronic band structure effects are investigated, leading to a $\mathrm{T} / \sinh (\mathrm{T})$ functional dependence. In Ref. [22] magnetic excitations (thermal spin waves) have been discussed as the main source of the T-dependence of $J_{\text {inter }}$. This leads to a power law in reduced temperature $T / T_{C}$ with a $3 / 2$ exponent. On the other hand, B. Heinrich recently discusses some experiments [23] and favors a linear T-dependence. To discriminate between this various analytical functions, experimental data over a large range in temperature are needed. But which T-range is relevant? The absolute range in degree Kelvin [23] is less relevant. More important seems to
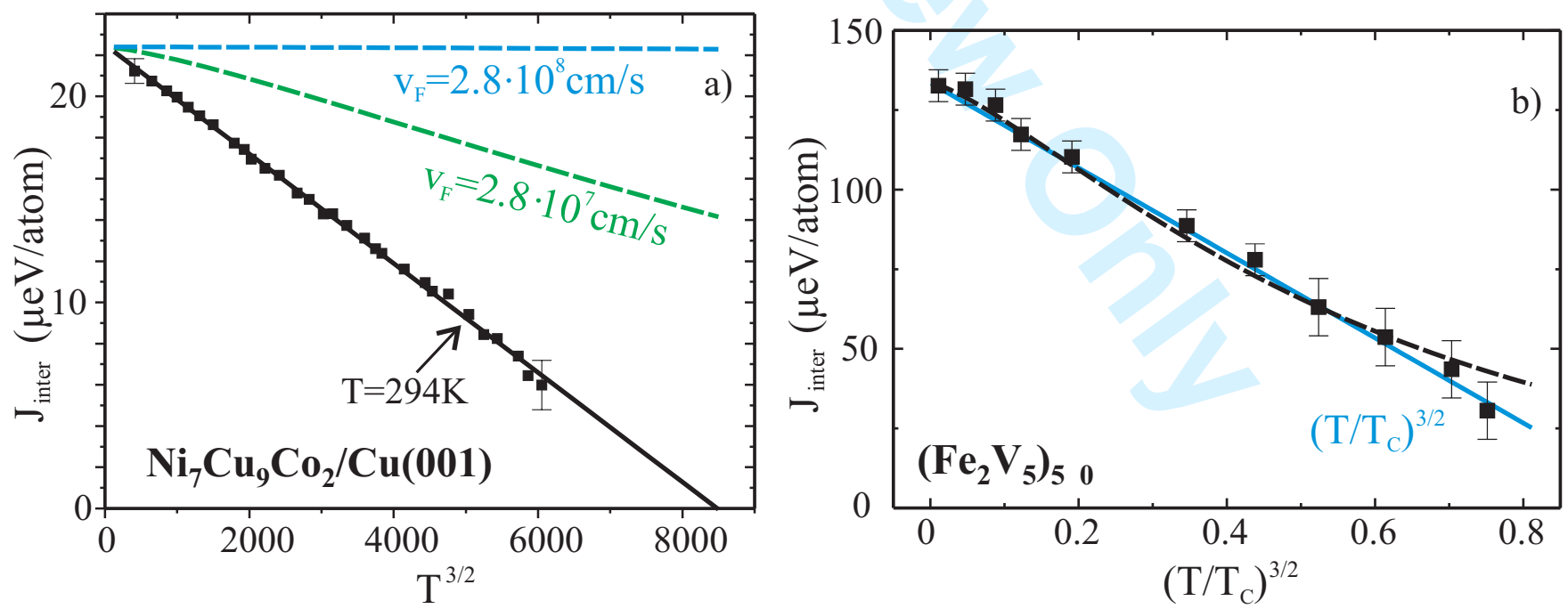

Figure 7. (color online) $J_{\text {inter }}$ as function of temperature. In a) the trilayer was measured to $\approx 400 \mathrm{~K}$, close to $T_{C}$. In b) the data for the multilayer are shown as function of the reduced temperature $t$. Taken from [25].

us the reduced temperature range, that is to say, to measure from low temperatures up to $T_{C}$. And this 
is difficult for several ML of Fe and Co. With the highest temperature of $400 \mathrm{~K}$ for thicker Fe films [24], $t=T / T_{C}$ will be $\leq 0.4$. We know only about one experiment [25] in which more or less the full range of reduced temperature was used. These authors measured 3 different multi- and trilayer systems $(\mathrm{Ni} / \mathrm{Cu} / \mathrm{Co}$, $\mathrm{Ni} / \mathrm{Cu} / \mathrm{Ni}$, and an $\mathrm{Fe} / \mathrm{V}$ multilayer). The reduced temperature covers almost the total range of $0<t \leq 0.9$, (Fig.7). Both cases, Fig.6 a and b, show an almost perfect power law behavior with a $3 / 2$ exponent. The dashed lines are a simulation of Ref. [21] with different Fermi velocities, the same is shown in b) with the dashed line. In a more extensive calculation of the band structure effects one should try to use more than one Fermi vector and other details of the Fermi surface. The results in Fig.7 are suggesting that the spin wave excitations [22] are dominant.

Nolting and coworkers [26] discussed the electronic effects of the T-dependence in the frame of ab initio theory combined with Fermi liquid model, as well as in the quantum well picture. To treat collective magnetic excitations they used a microscopic Heisenberg model. In addition to the IEC, $J_{\text {inter }}$, also a $J_{\text {intra }}$ is important. This is the exchange coupling within one FM film, a measure also for $T_{C}$. Its realistic values range in the $\mathrm{meV}$ regime, whereas $J_{\text {inter }}$ scales in the $\mu \mathrm{eV}$ regime. To extract the effect of the magnetic contributions alone for different spacer thicknesses, $J_{\text {inter }}$ has to be normalized to the parameter $J_{0} \equiv$ $J_{\text {inter }}(T=0)$. This is shown in Fig.8 a and b. In a) $J_{0}$ is constant and weaker or stronger $J_{\text {intra }}$ are used. In b) $J_{\text {intra }}$ is kept constant, but a gentile $J_{0}$ with FM and AFM coupling is used. In all cases the authors of Ref. [26] came to the conclusion, that $J_{\text {inter }}(T)$ does not follow an exact $3 / 2$ power law - see Fig. 8 a and b. But one may want to describe the temperature dependence of the IEC with an "effective" power law with

$$
J(T) \approx 1-A T^{n}, \quad n \approx 1.5
$$

With this combined theoretical and experimental effect it seems to be evident - and also plausible - that spin wave excitations are the dominant effect for the effective reduction of the IEC at finite $T$. Theory has the advantage to switch on and off different mechanisms. That is shown in Fig. 8 , the dashed line shows the reduction of $J_{\text {inter }}$ by spacer effects only, and the full line with "spacer + spin waves" [27].
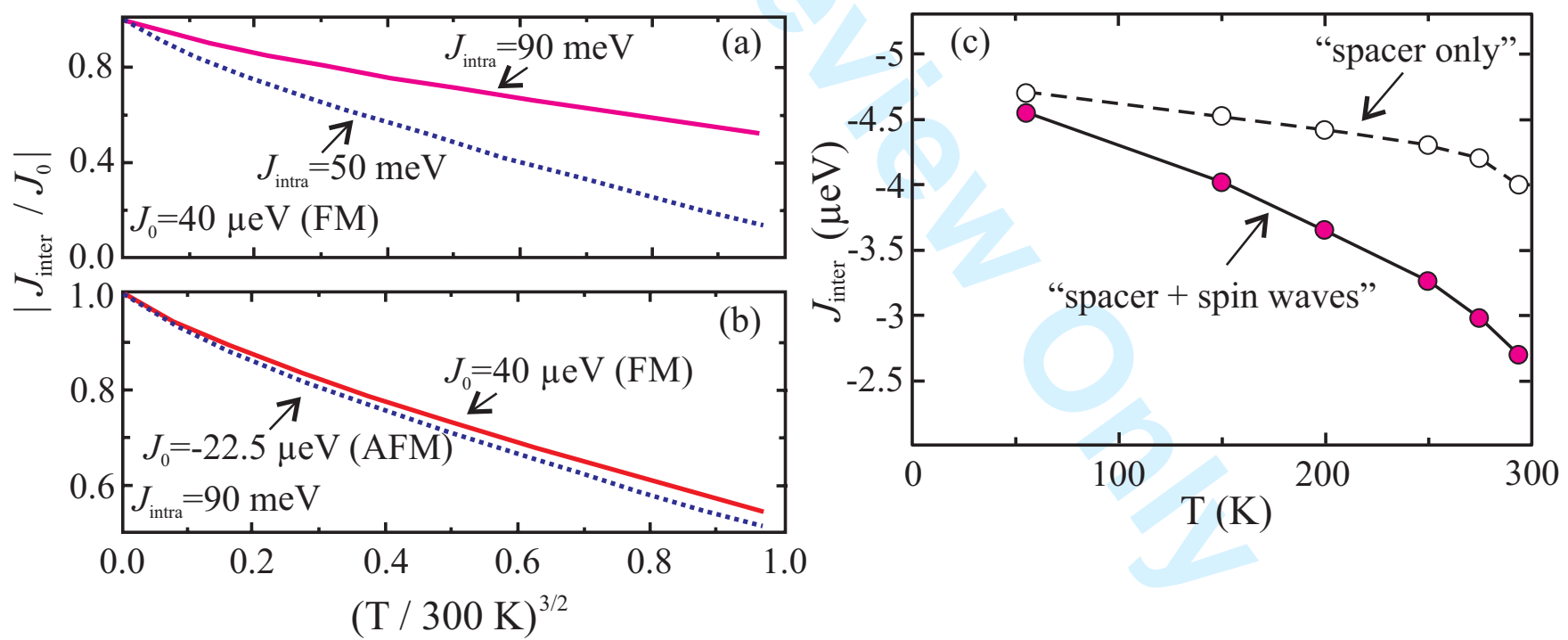

Figure 8. (color online) $J_{\text {inter }} / J_{0}$ vs. $T^{3 / 2}$ for a fixed $J_{0}$ in a) and for fixed $J_{\text {intra }}$ in b), from [26]. c) shows $J_{\text {inter }}$ as function of T, calculated with and without spin wave excitation [27].

That recent theoretical investigation in turn, inspired new FMR experiments [28]. The purpose was, to keep the two FM films constant and change only the spacer thickness $n$, and observe $J_{\text {inter }}$ as function of $n$, that should enter into the prefactor $A$ in Eq.2. What would we expect?

There are 3 possibilities: 
1. $A$ depends only on the interface $\Rightarrow A(d)=$ const

2. $A$ depends on electronic band structure $\Rightarrow A(d)=$ linear fct.

3. $A$ depends on spin waves, $\Rightarrow J_{\text {inter }} \Rightarrow A(d) \approx$ osc. fct.

To solve the question, the $\mathrm{Co} / \mathrm{Cu} / \mathrm{Ni}$ system, with $1.8 \mathrm{ML} \mathrm{Co}, n$ monolayers of $\mathrm{Cu}$ spacer, and 7 $\mathrm{ML} \mathrm{Ni}$ on a $\mathrm{Cu}(001)$ substrate was chosen [28]. This work provides for the first time an investigation of the temperature dependence of $J_{\text {inter }}$ entirely determined from the FMR angular dependence of the ferromagnetic resonance positions at each temperature for the $n=6 \mathrm{ML}$ film (Fig.9a). Obviously, the data do not follow a monotonic function of $d$, i. e. the slope $A$ for $\mathrm{n}=4$ fits between $\mathrm{n}=5$ and $\mathrm{n}=6$. The trend in Fig.9b is clear: Large $J_{0}$ produces a weak slope $A$ and vice versa, a very plausible result: The IEC and the thermal energy $k T$ are in competition. Very weak $J_{\text {inter }}$ allows easily thermal excitation of spin wave, and a stronger $J_{\text {inter }}$ reduces this effect.

Real interfaces will have steps and other imperfections. For magnetic and nonmagnetic ions at these sites the local DOS will be different from the bulk., i. e. $\mu_{S}$ may change, but more important $\mu_{L}$ will increase (see Sec.2). For a given geometrical arrangement and the corresponding interplay of $\mu_{S}$ and $\mu_{L}$ this will produce a unidirectional coupling, for example between a FM and AFM film. Such unidirectional coupling leads to, what is called "exchange bias". The $3 \times 3$ matrix of $J_{\text {inter }}{ }^{i j}$ may have no inversion symmetry, as discussed in $[1,2]$. Indeed the DM mechanism is employed again to explain "unidirectional exchange coupling" at interfaces.
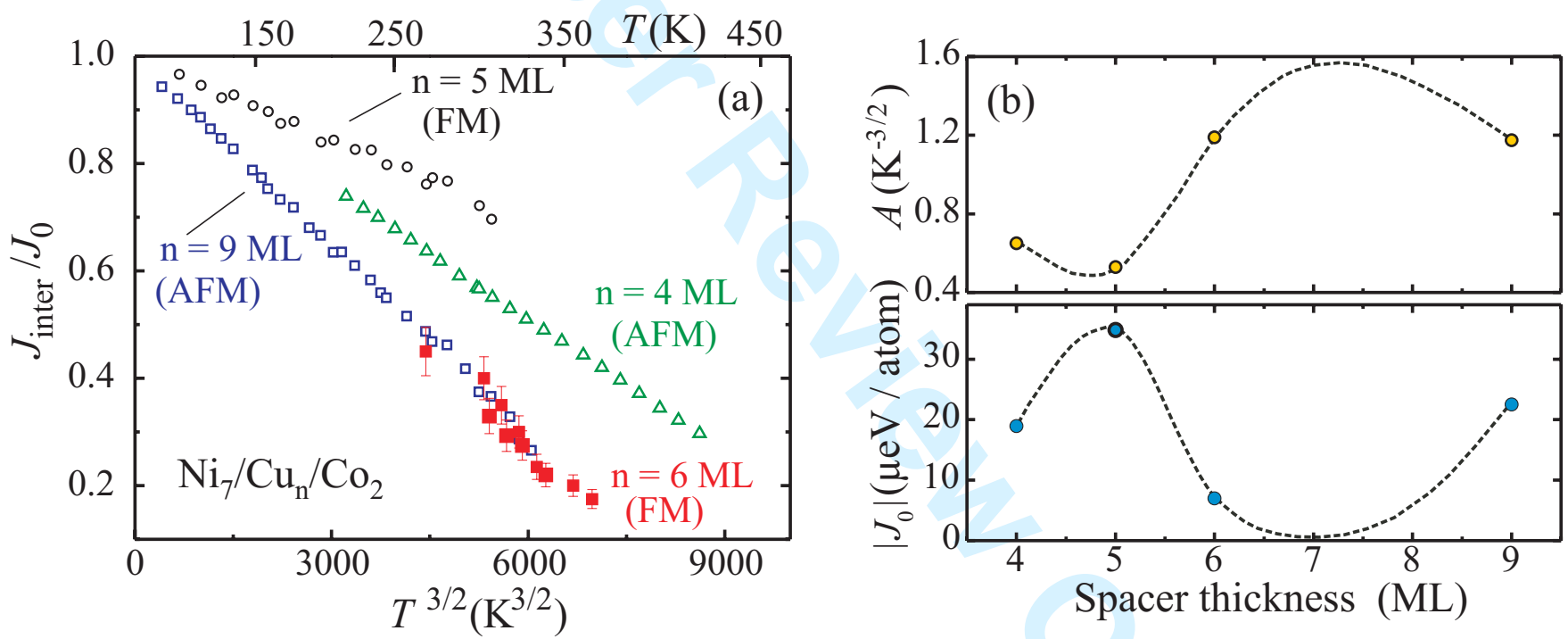

Figure 9. (color online) Normalized $J_{\text {inter }} \mathrm{vs} . \mathrm{T}^{1.5}$ for different spacer thickness $n$. The data points for the thicknesses of 4,5 and 9 ML were taken from Schwieger et al., [27], for details see [28].

\section{Conclusions}

Today's research on nanomagnetism, storage media, spin injection, etc. is very rich and successful. Many of the experimental findings are interpreted in a simple "spin-up, spin-down" picture, a common procedure in photo emission. The present contribution puts some emphasis on the fact that the orbital magnetic moment and angular momentum are crucial, they are not quenched, they originate the MAE. The anisotropy of $\mu_{L}$ is the leading ingredient to create an hysteresis loop with coercitive fields. Strictly speaking, not $S$ but $J$ is a good quantum number. In the past that has been demonstrated for Rare Earth spin glasses [29], simulating Ising or XY-systems. In some cases the $3 \times 3$ coupling matrix between two angular momentums 
has lower symmetry than uniaxial. This unidirectional mechanism with missing inversion symmetry, the DM interaction, is applicable to explain the "exchange bias" and it was successful to interpret the field cooling memory effect in spin glasses $[1,2]$. Finally, we show that for a joined interpretation by theory and experiment in nanomagnetism one must either extrapolate the experimental observables back to $T=0$, or include $T \neq 0$ in theory. In that case electronic band structure effects, smearing at the Fermi edge, seems to be a minor effect; most important are spin wave excitations and magnon-magnon scattering [30]. In Sec.2 we discussed the MAE and importance of orbital magnetism, in Sec.3 thermal spin wave excitations were added to understand $J_{\text {inter }}(\mathrm{T})$. Both steps are more or less "static arguments". For a real detailed microscopic understanding we need to consider in addition, that magnon-magnon scattering, spin-spin correlations are all important at interfaces of ultrathin ferromagnets. Some experiments and theoretical aspects, that not the static mean field picture but higher order spin correlations control nanomagnetism, are discussed in $[31,32]$.

\section{Acknowledgments}

We are very thankful to the theorists, who picked up our experimental findings and helped so for a more fundamental understanding of MAE and IEC in ultrathin ferromagnets. These are the groups of P. Weinberger, O. Eriksson and B. Johansson, D.L. Mills and R. Wu, K.-H. Bennemann and W. Nolting. All the experiments would not have been possible without my former coworkers: J. Lindner, K. Lenz, S. S. Kalarickal, E. Kosubek, X. Xu. In particular E. Kosubek and K. Lenz are acknowledged for assistance in preparing this manuscript. This work was supported in part by BMBF (05KS4 KEB/5) and DFG Sfb 658.

\section{References}

[1] A. Fert, Peter Levy, Phys. Rev. Lett. 44, 1538 (1980)

[2] Peter Levy, A. Fert, Phys. Rev. B 23, 4667 (1981)

[3] A. Ney, P. Poulopoulos and K. Baberschke Europhys. Lett. 54, 820 (2001)

[4] M. Farle, B. Mirwald-Schulz, A. Anisimov, W. Platow, and K. Baberschke Phys. Rev. B 55, 3708 (1997)

[5] K. Baberschke, M. Farle J. Appl. Phys. 81, 5038 (1997)

[6] A.N. Anisimov, M. Farle, P. Poulopoulos, W. Platow, K. Baberschke, P. Isberg, R. Wäppling, A.M.N. Niklasson and O. Eriksson Phys. Rev. Lett. 82, 2390 (1999)

[7] J. Lindner and K. Baberschke, J. Phys.: Condens. Matter 15, R193 and S465 (2003)

[8] Handbook of Magnetism and Advanced Magnetic Materials, K. Baberschke in Volume 3, p.1617 Edited by Helmut Kronmüller and Stuart Parkin, 2007 John Wiley \& Sons,

[9] O. Hjortstam, K. Baberschke, J.M. Wills, B. Johansson, and O. Eriksson Phys. Rev. B 55, 15026 (1997)

[10] B. Schulz and K. Baberschke, Phys. Rev. B 50, 13467 (1994)

[11] C. Uiberacker, J. Zabloudil, P. Weinberger, L. Szunyogh, C. Sommers, Phys. Rev. Lett. 82, 1289 (1999). Note that the relaxation of $-5.5 \%$ in this paper is normalized to $\mathrm{Ni}$ and identical to $-3.2 \%$ normalized to $\mathrm{Cu}$ in the present work.

[12] M. Farle, Rep. Prog. Physics 61, 755 (1998)

[13] J. Kohlhepp, H.J. Elmers, U. Gradmann, J. Magn. Magn. Mater. 121,487 (1993)

[14] F.J.A. den Broeder, W. Hoving, P.J.H. Bloemen, J. Magn. Magn. Mater. 191,562 (1991)

[15] M. Farle, W. Platow, E. Kosubek, K. Baberschke, Surf. Sci. 439,146 (1999)

[16] C.A.F. Vaz, J.A.C. Bland, G. Lauhoff, Rep. Prog. Phys.71,56501 (2008)

[17] R. Vollmer, Th. Gutjahr-Loser, J. Kirschner, S. von Dijken, B. Poelsma Phys. Rev. B 60, 6277 (1999)

[18] S. van Dijken, R. Vollmer, B. Poelsma, J. Kirschner J. Magn. Magn. Mater. 210,316 (2000)

[19] Jisang Hong, R.Q. Wu, J. Lindner, E. Kosubek, and K. Baberschke, Phys. Rev. Lett. 92, 147202 (2004)

[20] R. Hammerling, J. Zabloudil, P. Weinberger, J. Lindner, E. Kosubek, R. Nünthel, and K. Baberschke, Phys. Rev. B 68, 092406 (2003).

[21] P. Bruno, Phys. Rev. B 52, 411 (1995) and V. Drchal, J. Kudrnovsky, P. Bruno, I. Turek, P. H. Dederichs, P. Weinberger, Phys. Rev. B 60, 9588 (1999).

[22] N. S. Almeida, D. L. Mills, and M. Teitelman, Phys. Rev. Lett. 75, 733 (1995).

[23] B. Heinrich inMagnetic Heterostructures Edited by H. Zabel and S.D. Bader, Springer Tracts of Modern Physics 227, 185 (2008).

[24] Z. Celisnki, B. Heinrich, J.F. Cochran, J. Magn. Magn. Mater. 145, L1 (1995)

[25] J. Lindner, C. Rüdt, E. Kosubek, P. Poulopoulos, K. Baberschke, P. Blomquist, R. Wäppling, and D. L. Mills, Phys. Rev. Lett. 88, 167206 (2002).

[26] S. Schwieger, W. Nolting, Phys. Rev. B 69, 224413 (2004)

[27] S. Schwieger, J. Kienert, K. Lenz, J. Lindner, K. Baberschke, and W. Nolting, Phys. Rev. Lett. 98, 057205 (2007) and J. Magn. Magn. Mater. 310, 2301 (2007).

[28] S. S. Kalarickal, X. Xu, K. Lenz, W. Kuch, K. Baberschke, Phys. Rev. B 75, 224429 (2007).

[29] K. Baberschke, P. Pureur, A. Fert, R. Wendler, S. Senoussi, Phys. Rev. B 29, 4999 (1984)

[30] J. Lindner, K. Lenz, E. Kosubek, K. Baberschke, D. Spoddig, R. Meckenstock, J. Pelzl, Z. Frait and D.L. Mills, Phys. Rev. B 68, $060102(\mathrm{R})(2003)$.

[31] K. Baberschke, Physica status solidi (b) 245, 174 (2008).

[32] L. Bergqvist, O. Eriksson , J. Phys.: Cond. Matter 18, 4853 (2006) 
(a)

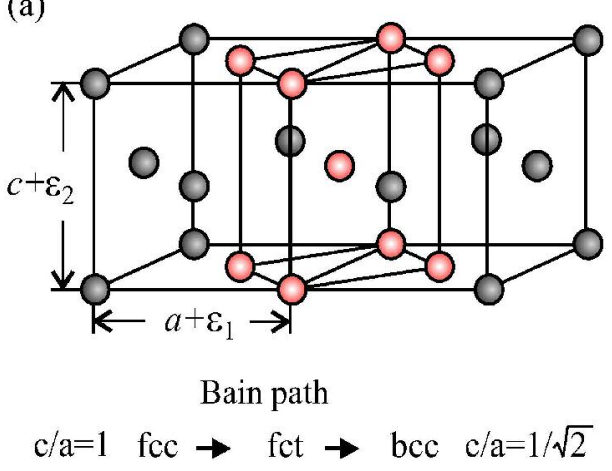

(b)

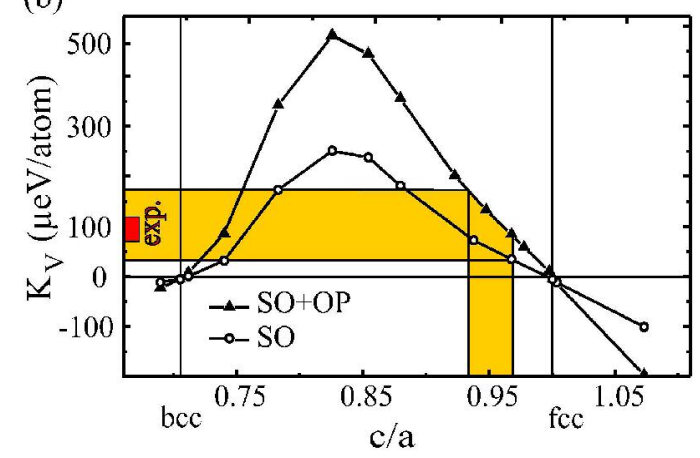

$117 \times 43 \mathrm{~mm}(600 \times 600 \mathrm{DPI})$ 
(a)

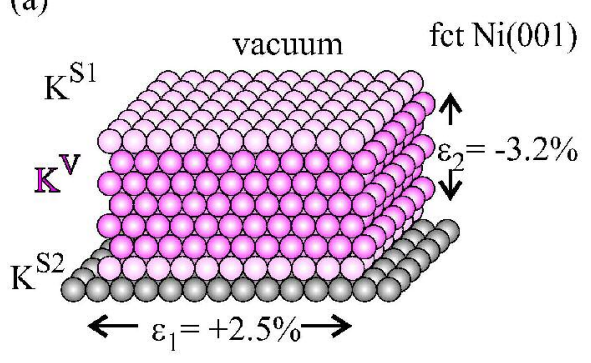

fcc $\mathrm{Cu}(001)$ substrate $\left(\mathrm{a}_{\mathrm{p}}=2.55 \AA\right)$

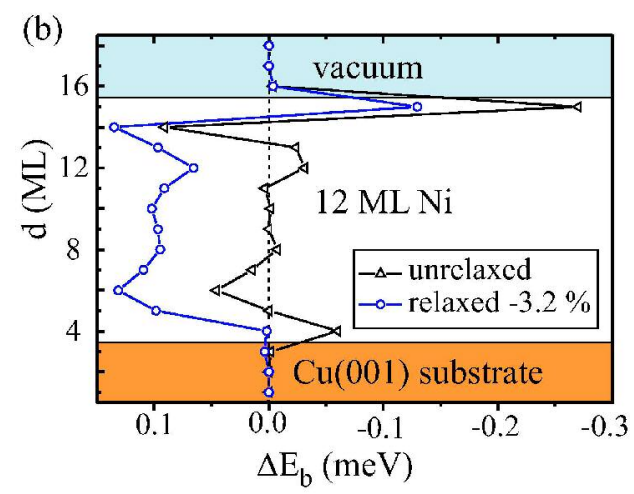

$117 \times 45 \mathrm{~mm}(600 \times 600 \mathrm{DPI})$ 

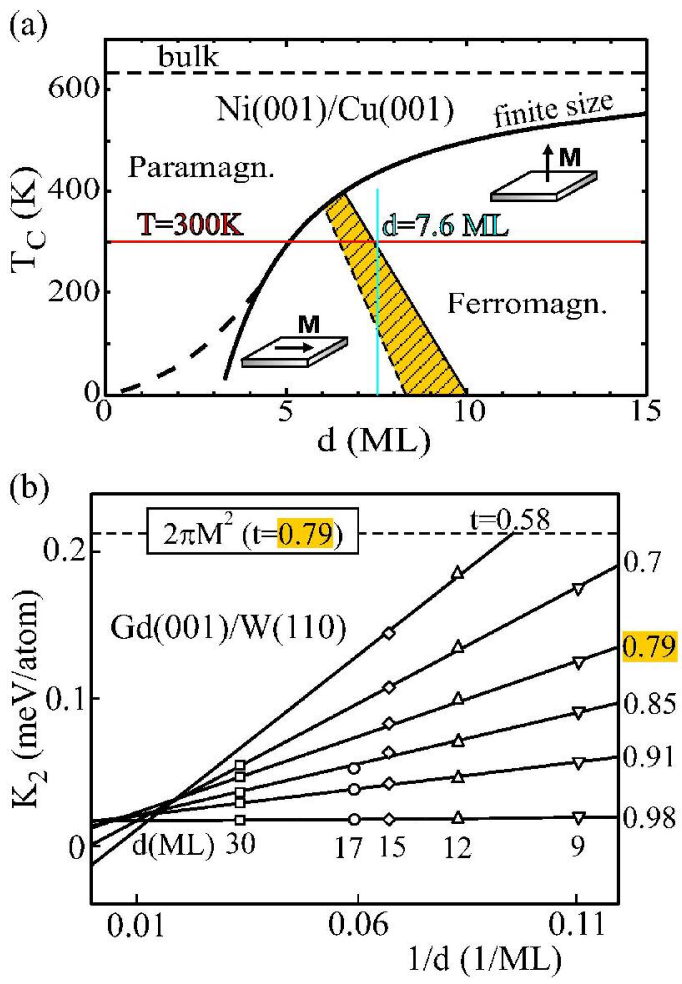

(c)

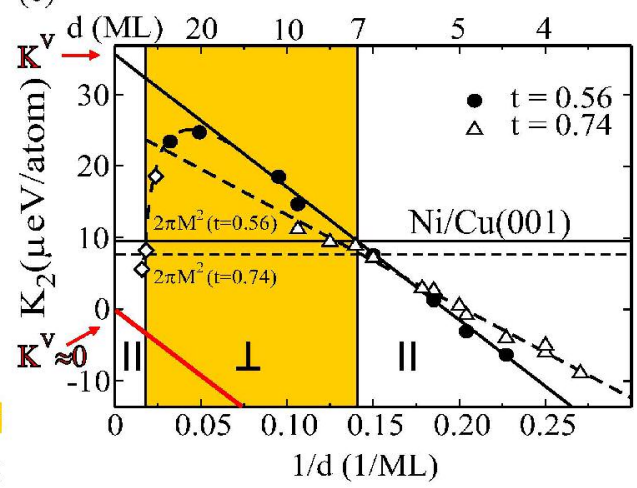

$117 \times 88 \mathrm{~mm}(600 \times 600 \mathrm{DPI})$ 


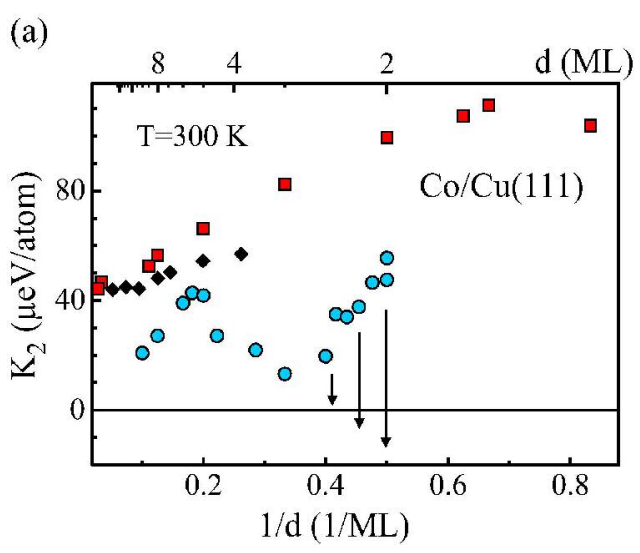

(b)

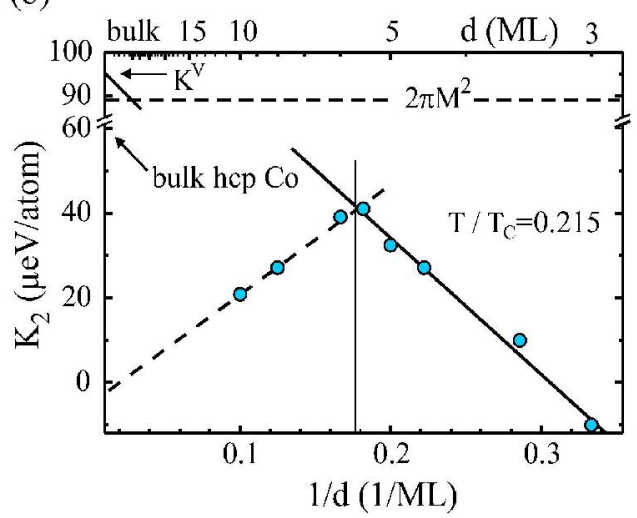

$118 \times 49 \mathrm{~mm}(600 \times 600 \mathrm{DPI})$ 


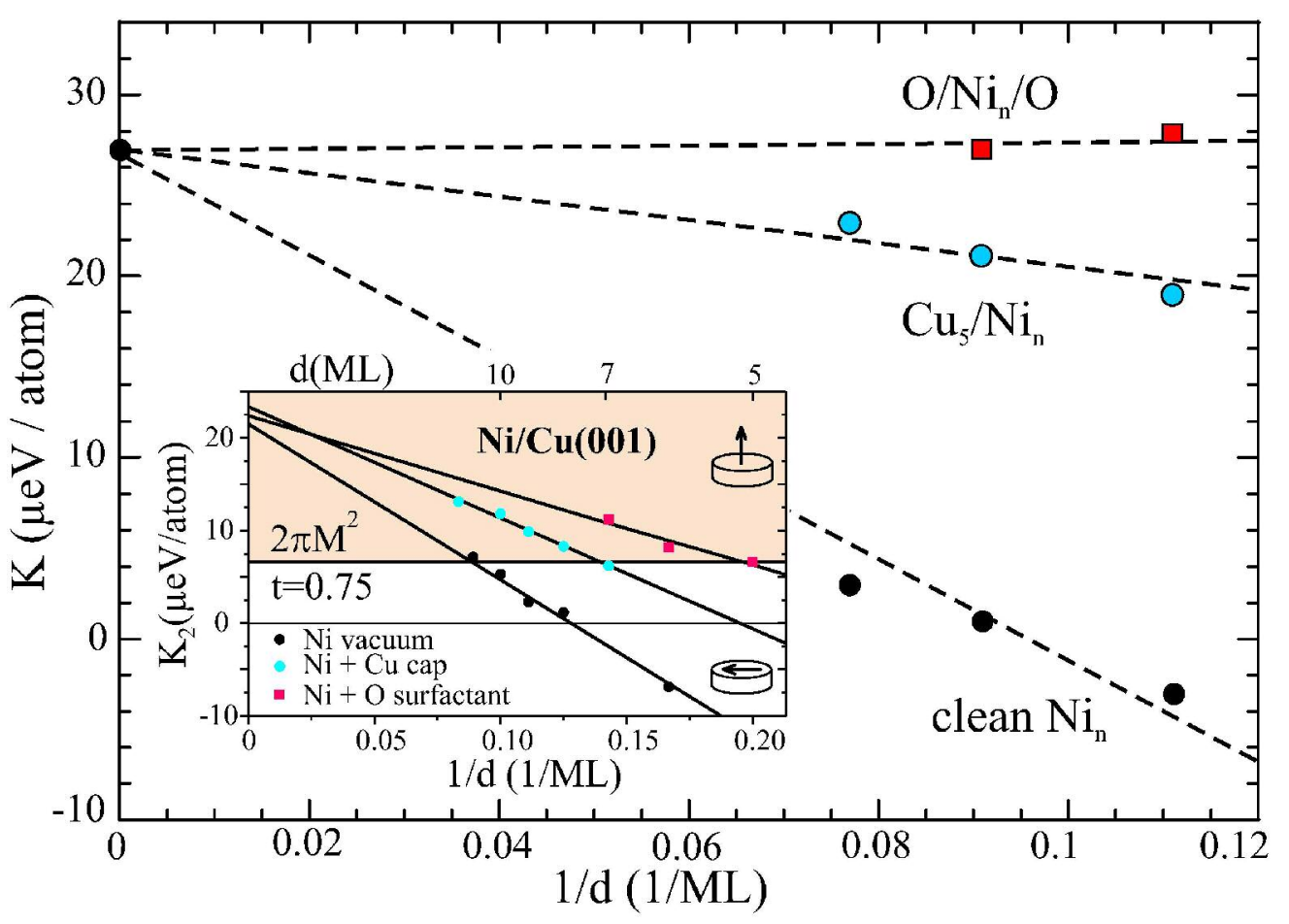

$116 \times 83 \mathrm{~mm}(600 \times 600 \mathrm{DPI})$ 


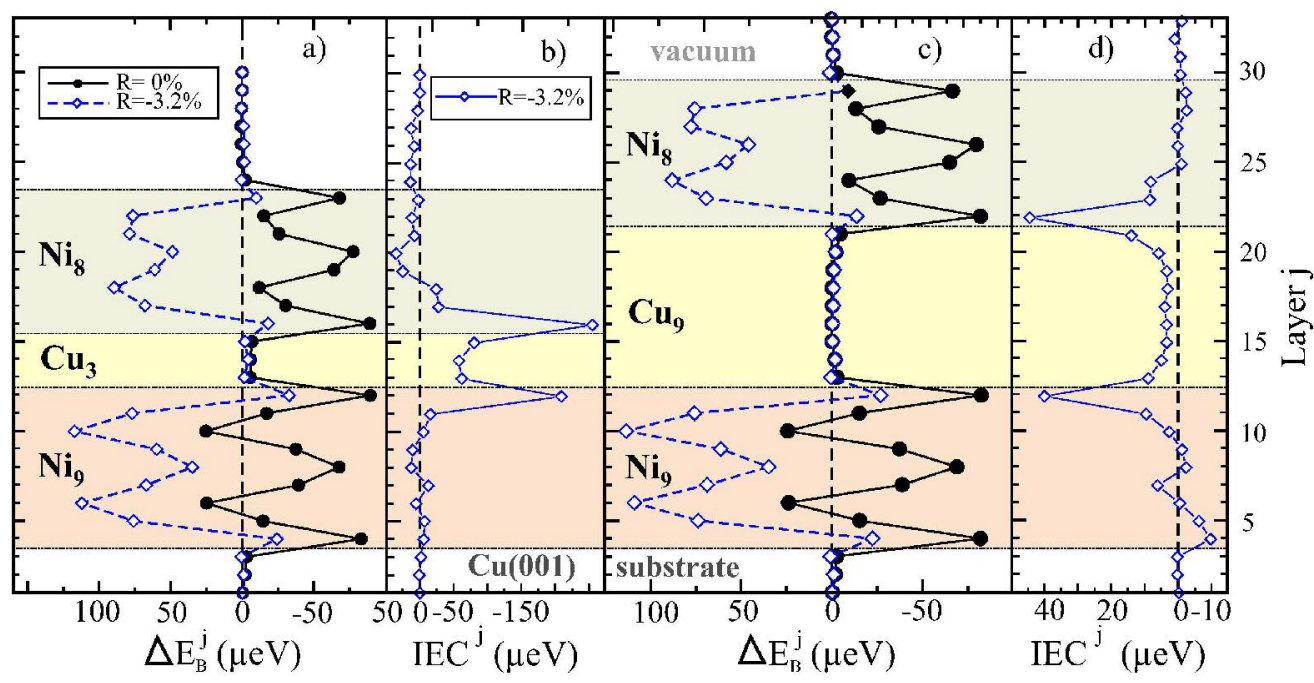

$116 \times 71 \mathrm{~mm}(600 \times 600 \mathrm{DPI})$ 

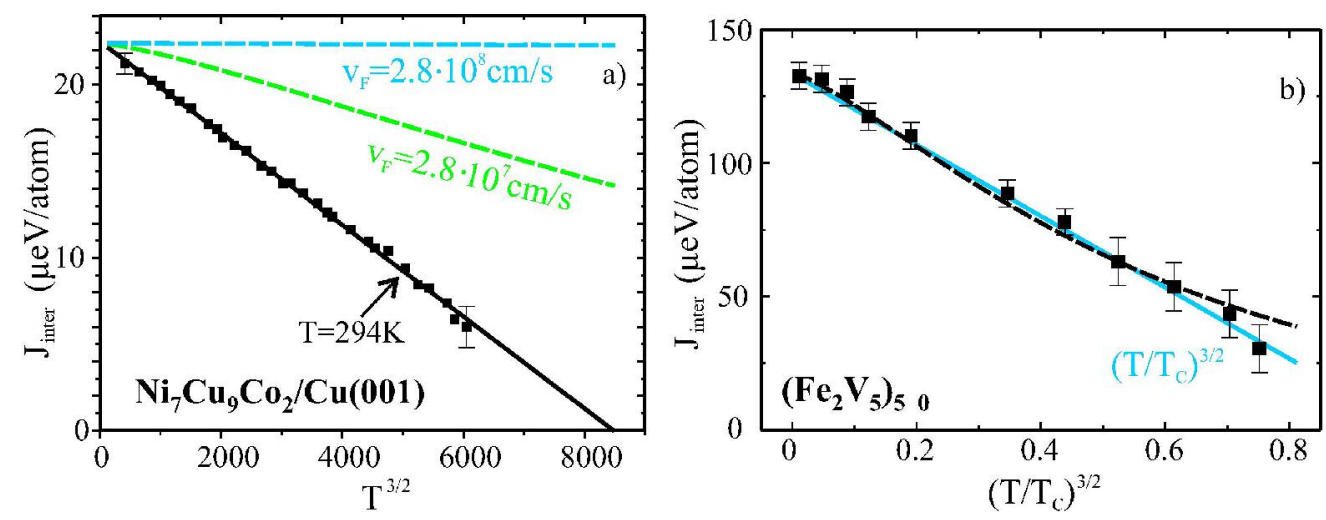

$117 \times 44 \mathrm{~mm}(600 \times 600 \mathrm{DPI})$ 

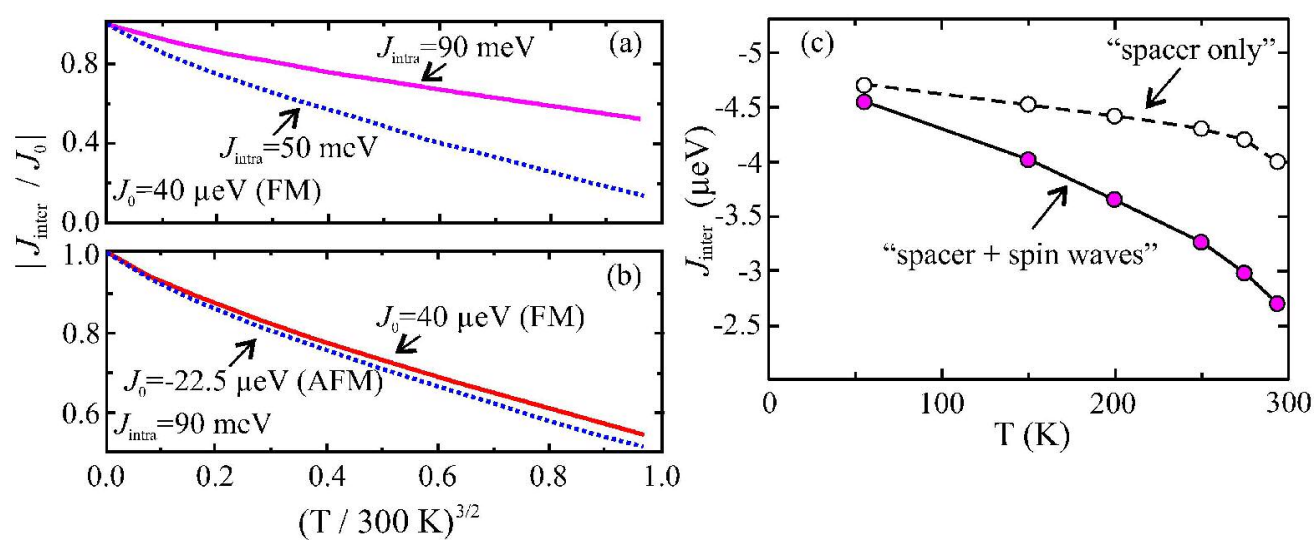

$119 \times 48 m m(600 \times 600$ DPI $)$ 

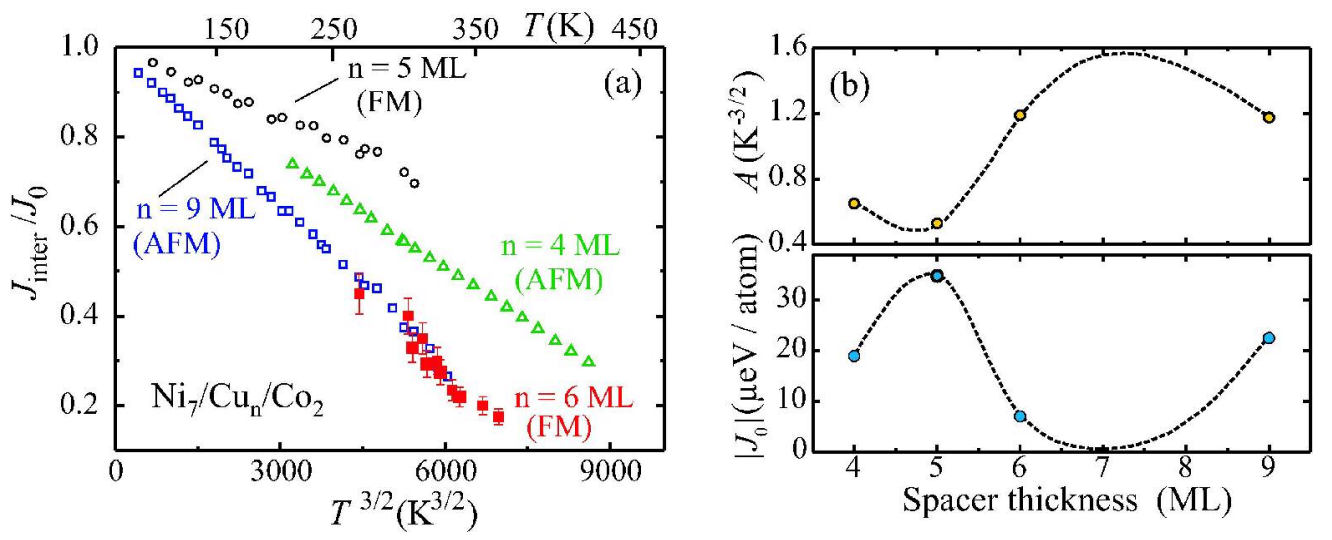

$117 \times 47 m m(600 \times 600$ DPI $)$ 